\title{
Towards a comprehensive knowledge of the open cluster Haffner 9
}

\author{
Andrés E. Piatti ${ }^{1,2 \star}$ \\ ${ }^{1}$ Observatorio Astronómico, Universidad Nacional de Córdoba, Laprida 854, 5000, Córdoba, Argentina \\ ${ }^{2}$ Consejo Nacional de Investigaciones Científicas y Técnicas, Av. Rivadavia 1917, C1033AAJ, Buenos Aires, Argentina
}

Accepted XXX. Received YYY; in original form ZZZ

\begin{abstract}
We turn our attention to Haffner 9, a Milky Way open cluster whose previous fundamental parameter estimates are far from being in agreement. In order to provide with accurate estimates we present high-quality Washington $C T_{1}$ and Johnson $B V I$ photometry of the cluster field. We put particular care in statistically clean the colourmagnitude diagrams (CMDs) from field star contamination, which was found a common source in previous works for the discordant fundamental parameter estimates. The resulting cluster CMD fiducial features were confirmed from a proper motion membership analysis. Haffner 9 is a moderately young object (age $\sim 350 \mathrm{Myr}$ ), placed in the Perseus arm -at a heliocentric distance of $\sim 3.2 \mathrm{kpc}-$, with a lower limit for its present mass of $\sim 160 \mathrm{M}_{\odot}$ and of nearly metal solar content. The combination of the cluster structural and fundamental parameters suggest that it is in an advanced stage of internal dynamical evolution, possibly in the phase typical of those with mass segregation in their core regions. However, the cluster still keeps its mass function close to that of the Salpeter's law.
\end{abstract}

Key words: techniques: photometric - Galaxy: open clusters and associations: general.

\section{INTRODUCTION}

Different statistical procedures have been proposed with an acceptable success, in order to avoid as much as possible the field contamination in cluster colour-magnitude diagrams (CMDs) analysis (e.g. Bonatto \& Bica 2007; Pavani \& Bica 2007; Maia et al. 2010) The developed statistical methods basically involve: (i) dividing the full range of magnitude and colour of a given CMD into a grid whose cells have axes along the magnitude and colour directions, (ii) computing the expected number-density of field stars in each cell based on the number of comparison field stars with magnitude and colour compatible with those of the cell, and (iii) subtracting randomly the expected number of field stars from each cell. Although the methods reapply the cleaning procedure using different cell sizes in the CMDs, they are fixed each time, i.e., they do not vary across the CMDs.

From our experience in cleaning the field star contamination in the cluster CMDs, we have identified some situations which still need our attention. It frequently happens that some parts of the CMDs are more populated than others, so that fixing the size of the cells in the CMDs becomes

* E-mail: andres@oac.unc.edu.ar a difficult task. Small cells do not usually carry out a satisfactory job in CMD regions with a scarce number of fields stars, while big cells fail in populous CMD regions. Thus, relatively bright field red giants with small photometric errors could not be subtracted and, consequently, the cluster CMD could show spurious red giant features. A compromise between minimizing the residuals left after the subtractions of field stars from the cluster CMDs and maximizing the cleaning of field stars is always desiderable.

In this paper, we present a comprehensive multi-band photometric analysis of Haffner 9 from $B V I$ and Washington $C T_{1}$ photometry. The cluster has been previously studied from 2MASS and BVI photometry. However, a relatively lower photometric accuracy and shallower limited magnitude, in combination with a not much pinpointed treatment of field decontamination led those studies to discordant results. In Section 2 we describe the collection and reduction of the available photometric data and their thorough treatment in order to build a extensive and reliable data set. The cluster structural and fundamental parameters are derived from star counts and colour-magnitude and colour-colour diagrams, respectively, as described in Section 3. The analysis of the results of the different astrophysical parameters obtained is carried out in Section 4, where implications about 
the stage of its dynamical evolution are suggested. Finally, Section 5 summarizes the main conclusion of this work.

\section{DATA COLLECTION AND REDUCTION}

We used CCD images ${ }^{1}$ obtained on the nights of December $18^{\text {th }}$ and $19^{\text {th }}, 2004$ with a $2048 \times 2048$ pixel Tektronix CCD attached to the $0.9 \mathrm{~m}$ telescope (scale $0.396 \mathrm{arcsec} / \mathrm{pixel}$ ) at Cerro Tololo Inter-American Observatory (CTIO, Chile). Its field of view is $13.6 \times 13.6 \mathrm{arcmin}^{2}$. We used the Washington $C$ (Canterna 1976) and Kron-Cousins $R$ filters. The latter has a significantly higher throughput as compared with the standard Washington $T_{1}$ filter so that $R$ magnitudes can be accurately transformed to yield $T_{1}$ magnitudes Geisler (1996). We used a series of bias, dome and sky flat-field exposures per filter to calibrate the CCD instrumental signature. We also utilised images for the Small Magellanic Cloud (SMC) cluster Lindsay 106, which was previously observed at La Silla (ESO, Chile) with the $C$ and $T_{1}$ filters (Piatti et al. 2007). Lindsay 106 was used here only as control cluster, i.e., to verify the quality of the present CTIO photometry. Table 1 shows the log of the observations with filters, exposure times, airmasses and seeing estimates. A large number (typically 20) of standard stars from the list of Geisler (1996) was also observed on each night. They cover wide colour and airmass ranges, so that we could calibrate properly the program stars observed on these nights.

The stellar photometry was performed using the star finding and point spread function (PSF) fitting routines in the DAOPHOT/ALLSTAR suite of programs (Stetson et al. 1990). Radially varying aperture corrections were applied to take out the effects of PSF variations across the field of view, although a quadratically varying PSF was employed. The resultant instrumental magnitudes were standardized using the equations:

$$
\begin{array}{r}
c=(3.679 \pm 0.021)+T_{1}+\left(C-T_{1}\right)+(0.294 \pm 0.014) \times X_{C} \\
-(0.085 \pm 0.005) \times\left(C-T_{1}\right),
\end{array}
$$

$$
\begin{array}{r}
r=(3.206 \pm 0.021)+T_{1}+(0.115 \pm 0.014) \times X_{R} \\
-(0.014 \pm 0.004) \times\left(C-T_{1}\right),
\end{array}
$$

where $X$ represents the effective airmass. Capital and lowercase letters stand for standard and instrumental magnitudes, respectively. The coefficients were derived through the IRAF $^{2}$ routine FITPARAM. The root mean square (rms) deviations of the fitted values from the fits to the standards were 0.021 for $c$ and 0.014 for $r$, which indicates that the nights were photometric. We combined all the independent measurements using the stand-alone DAOMATCH

1 The images are made available to the public through http://www.noao.edu/sdm/archives.php, SMARTS Consortium, DDT, PI: Clariá.

2 IRAF is distributed by the National Optical Astronomy Observatories, which is operated by the Association of Universities for Research in Astronomy, Inc., under contract with the National Science Foundation

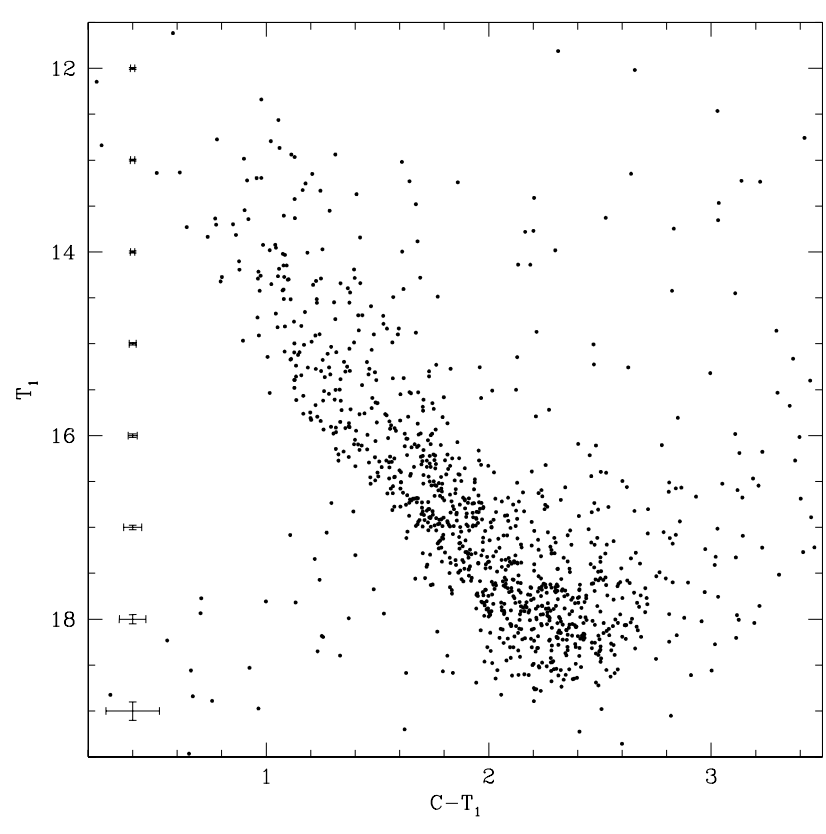

Figure 1. Observed CMD for all the stars measured in the field of haffner 9. Errorbars at the left-hand margin represent the photometric uncertainties given by DAOPHOT.

and DAOMASTER programmes, kindly provided by Peter Stetson. The final information gathered for each cluster consists of a running number per star, the $x$ and $y$ coordinates, the measured $T_{1}$ magnitudes, $C-T_{1}$ colours, and the observational errors $\sigma\left(T_{1}\right)$ and $\sigma\left(C-T_{1}\right)$. In the case of Haffner 9, for which we combined respectively four different photometric tables, we also included the number of measures performed in $T_{1}$ and $C-T_{1}$. Table 2 gives this information, where only a portion of it is shown for guidance regarding its form and content. The whole content of Table 2, as well as that for Lindsay 106 (Table 3), is available in the online version of the journal.

The calculated internal accuracy of the photometry has been computed according to the criteria given by Stetson et al. (1990) and includes random noise, errors in the modelling and centering of the stellar profile. These internal standard errors provided by DAOPHOT for the $T_{1}$ magnitude and $C-T_{1}$ colour have been represented by errorbars at the left-hand margin of the observed CMD in Fig. 1. Fig. 2 shows how the differences between our $T_{1}$ magnitudes and $C-T_{1}$ colours and those obtained by Piatti et al. (2007) vary as a function of $T_{1}$. Offsets of $\Delta T_{1}=T_{1_{\text {pub }}}-T_{1_{\text {thiswork }}}=-0.055$ \pm 0.092 and $\Delta\left(C-T_{1}\right)=\left(C-T_{1}\right)_{\text {pub }}-\left(C-T_{1}\right)_{\text {thiswork }}=$ $0.050 \pm 0.112$ have been computed from 425 stars measured in common.

\section{1 $B V I$ photometric data sets}

Carraro et al. (2013, hereafter C13) have carried out a photometric $B V I$ campaign focused on the study of open clusters preferentially located towards the Third Galactic Quadrant. They used the Y4KCAM camera attached to the CTIO 1-m telescope, operated by the SMARTS consortium during an observation run in 2005 November-December. In the afore- 
Table 1. Observations log of the open cluster Haffner 9 and the control field Lindsay 106.

\begin{tabular}{|c|c|c|c|c|c|c|c|c|c|}
\hline Cluster $^{a}$ & $\begin{array}{l}\text { R.A. } \\
(\text { h m s) }\end{array}$ & $\begin{array}{l}\text { Dec. } \\
\left(0,{ }^{\circ}, \prime \prime\right)\end{array}$ & $\begin{array}{c}l \\
\left(^{\circ}\right)\end{array}$ & $\begin{array}{l}\mathrm{b} \\
\left(^{\circ}\right)\end{array}$ & date & filter & $\begin{array}{c}\text { exposure } \\
\text { (sec) }\end{array}$ & airmass & $\begin{array}{c}\text { seeing } \\
\left({ }^{\prime \prime}\right)\end{array}$ \\
\hline \multirow[t]{2}{*}{ Lindsay 106, ESO 29-SC44 } & 13038 & -760316 & 299.82 & -40.84 & Dec. 19 & $C$ & 2400 & 1.47 & 1.4 \\
\hline & & & & & & $R$ & 900 & 1.45 & 1.3 \\
\hline \multirow[t]{6}{*}{ Haffner 9} & 72442 & $\begin{array}{lll}-17 & 00 & 10\end{array}$ & 231.80 & -0.59 & Dec. 18 & $C$ & 300 & 1.05 & 1.3 \\
\hline & & & & & & $C$ & 300 & 1.05 & 1.3 \\
\hline & & & & & & $R$ & 10 & 1.06 & 1.1 \\
\hline & & & & & & $R$ & 10 & 1.06 & 1.1 \\
\hline & & & & & & $R$ & 30 & 1.07 & 1.2 \\
\hline & & & & & & $R$ & 30 & 1.07 & 1.2 \\
\hline
\end{tabular}

${ }^{a}$ Cluster identifications are from Lindsay $(1958, \mathrm{~L})$ and Lauberts $(1982, \mathrm{ESO})$.

Table 2. $C T_{1}$ data of stars in the field of Haffner 9.

\begin{tabular}{lcccccccc}
\hline Star & $\begin{array}{c}x \\
(\text { pixel })\end{array}$ & $\begin{array}{c}y \\
(\text { pixel })\end{array}$ & $\begin{array}{c}T_{1} \\
(\mathrm{mag})\end{array}$ & $\begin{array}{c}\sigma\left(T_{1}\right) \\
(\mathrm{mag})\end{array}$ & $\mathrm{n}_{T_{1}}$ & $\begin{array}{c}C-T 1 \\
(\mathrm{mag})\end{array}$ & $\begin{array}{c}\sigma\left(C-T_{1}\right) \\
(\mathrm{mag})\end{array}$ & $\mathrm{n}_{C-T_{1}}$ \\
\hline- & - & - & - & - & - & - & - & - \\
102 & 1613.207 & 182.751 & 14.554 & 0.015 & 4 & 1.228 & 0.059 & 4 \\
103 & 608.814 & 186.716 & 16.886 & 0.019 & 4 & 1.739 & 0.045 & 4 \\
104 & 1012.074 & 193.898 & 15.197 & 0.014 & 4 & 1.350 & 0.029 & 4 \\
- & - & - & - & - & - & - & - & - \\
\hline
\end{tabular}

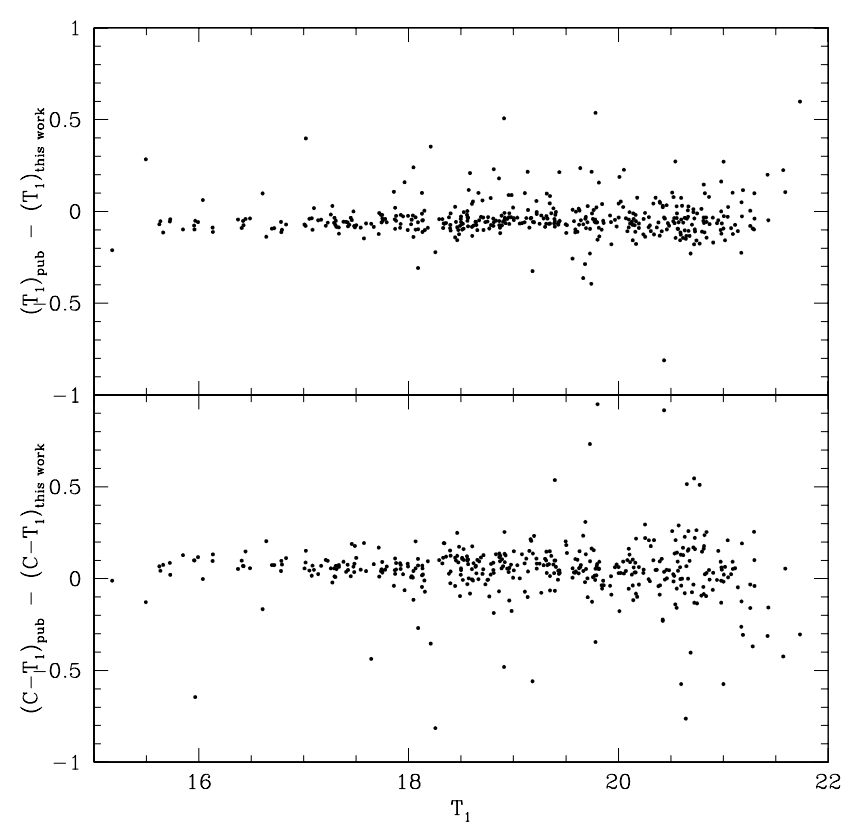

Figure 2. Comparison of $C T_{1}$ photometry between that of Piatti et al. (2007) and this work for the control cluster Lindsay 106.

mentioned work they did not include Haffner 9, although they made their $B V I$ photometry publicly available ${ }^{3}$.

The camera used to obtain those data was equipped with an STA $4064 \times 4064$ CCD with $15-\mu$ m pixels, yielding a scale of $0.289^{\prime \prime} /$ pixel and a field-of-view (FOV) of $20^{\prime} \times 20^{\prime}$. The CCD was operated without binning, at a nominal gain

3 VizieR On-line Data Catalog: J/MNRAS/428/502 of $1.44 \mathrm{e}-/ \mathrm{ADU}$, implying a readout noise of 7 e- per quadrant (four amplifiers). Their available photometry add significant value to our study, because of the larger FOV and the fainter magnitude limit reached using a different telescope/photometric system setup. We refer the reader to the work by $\mathrm{C} 13$ for details concerning the data processing, the measurement of photometric magnitudes and the standardization of their photometry to the $B V I$ system.

We compared Carraro et al. (2013)'s photometry with that obtained by Hasegawa et al. (2008, hereafter H08) and concluded that the former needs to be corrected by :

$V_{\mathrm{std}}=V_{\mathrm{C} 13}-0.9$

$B_{\mathrm{std}}=(B-V)_{\mathrm{C} 13} * 0.74+0.4$

$I_{\mathrm{std}}=-(V-I)_{\mathrm{C} 13} * 0.87+$ V carr $* 0.05+0.45$

in order to match the latter. As for the H08 photometry, we used the ridgelines in their CMDs -thought to be the mean locus of the fiducial cluster sequence-, since the data are not available from the authors.

We finally merged our Washington $C T_{1}$ and the corrected available $B V I$ data sets into only one master table, which we use in our subsequent an analysis.

\section{ANALYSIS OF THE PHOTOMETRIC DATA}

The pipeline followed in the analysis of the present photometric data sets involved: i) define the cluster central coordinates and trace its stellar density radial profile; ii) derive the cluster structural parameters; iii) decontaminate the cluster CMD from field stars and; iv) estimate the cluster fundamental parameters. 


\subsection{Cluster extension}

In order to obtain the stellar density radial profile of Haffner 9, we started by estimating its geometrical centre. We did that by fitting Gaussian distributions to the star counts in the $x$ and $y$ directions. The number of stars projected along the $x$ and $y$ directions were counted within intervals of $20,40,60,80,100$ pixel wide, and the Gaussian fits repeated each time. The fits of the Gaussians were performed using the NGAUSSFIT routine in the STSDAS/IRAF package. We adopted a single Gaussian and fixed the constant to the corresponding background levels (i.e. stellar field density assumed to be uniform) and the linear terms to zero. The centre of the Gaussian, its amplitude, and its FWHM acted as variables. Finally, we averaged the five different Gaussian centres with a typical standard deviation of \pm 40 pixels $\left( \pm 16.0^{\prime \prime}\right)$.

The estimated geometrical centre was then used to built the cluster stellar density profile from star counts performed within boxes of 60 pixels per side distributed throughout the whole observed field. The chosen box size allowed us to statistically sample the stellar spatial distribution. Thus, the number of stars per unit area at a given radius $r$ can be directly calculated through the expression:

$\left(n_{r+30}-n_{r-30}\right) /\left(m_{r+30}-m_{r-30}\right)$,

where $n_{r}$ and $m_{r}$ represent the number of stars and boxes, respectively, included in a circle of radius $r$. The advantage of this method over the frequent counting of stars in annular regions around the cluster centre relies on the fact that is not required a complete circle of radius $r$ within the observed field to compute the mean stellar density at that distance. Therefore, it is possible to estimate the background level with high precision using regions located far away from the cluster centre. With a good placement of the background level, the cluster radius $\left(r_{c l s}\right)$ results, in turn, in a more reliable estimate. Fig. 3 shows with open and filled circles the observed and background subtracted density profiles expressed as number of stars per $\operatorname{arcsec}^{2}$-, respectively, while the errobars represent the rms errors. In the case of the background subtracted density profile we added the mean error of the background star counts. The background level and the cluster radius $\left(r_{c l s}=200_{-40}^{+50}\right.$ arcsec $)$ are indicated by solid horizontal and vertical lines, respectively; their uncertainties are in dotted lines.

We fitted the background corrected density profile using both King (1962) and Plummer (1911) models in order to get independent estimates of the cluster core $\left(r_{c}\right)$, half-mass $\left(r_{h}\right)$ and tidal $\left(r_{t}\right)$ radii, respectively. We used a grid of $r_{c}$, $r_{h}$ and $r_{t}$ values spanning the known range of radii of open clusters (Piskunov et al. 2007) and minimised $\chi^{2}$. We finally derived $r_{c}=70 \pm 10$ arcsec, $r_{h}=125 \pm 15$ arcsec and $r_{t}$ $=400 \pm 100$ arcsec, respectively. In Fig. 3 we superimposed the respective King's and Plummer's curves with blue and oorange solid lines, respectively.

\subsection{Field star decontamination}

Because Fig. 1 reveals that both cluster and field star sequences are more or less superimposed, we must firstly separate the cluster stars from those belonging to the surround-

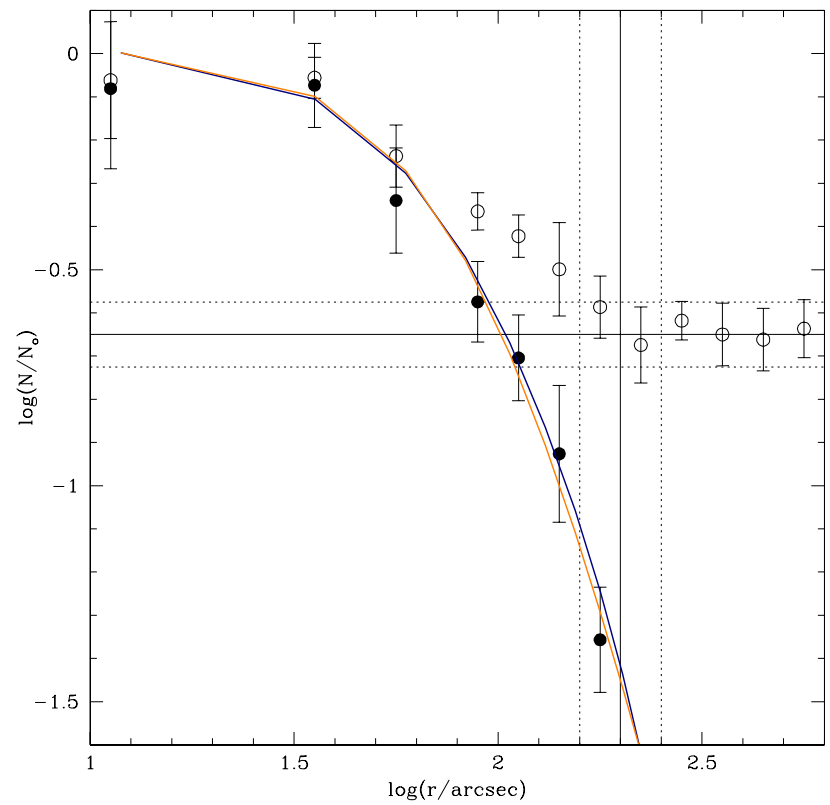

Figure 3. Stellar density profile obtained from star counts. Open and filled circles refer to measured and background subtracted density profiles, respectively. Blue and orange solid lines depict the fitted King and Plummer curves, respectively.

ing fields on a statistical basis in order to meaningfully use that CMD to estimate the cluster parameters. Note that both cluster and field stars are affected by nearly the same interstellar reddening, which is indeed what causes the overlapping of their main sequences (MSs). This fact makes the cleaning of the cluster CMD even more challenging.

To filter the field stars from the CMDs, we applied a statistical procedure which consists, firstly, in adopting two CMDs from different regions located far from the cluster. The dimension of each selected field region was $\pi r_{c l s}{ }^{2}$ and acted as references to statistically filter an equal circular area centred on Haffner 9.

Secondly, by starting with reasonably large boxes - typically $\left(\Delta\left(T_{1}\right), \Delta\left(C-T_{1}\right)\right)=(1.00,0.50) \mathrm{mag}$ - centred on each star in both field CMDs and by subsequently reducing their sizes until they reach the stars closest to the boxes' centres in magnitude and colour, separately, we defined boxes which result in use of larger areas in field CMD regions containing a small number of stars, and vice versa. Note that the definition of the position and size of each box involves two field stars, one at the centre of the box and another -the closest one to box centre - placed on the boundary of that box. Piatti \& Bica (2012) have shown that this is an effective way of accounting for the local field-star signature in terms of stellar density, luminosity function and/or colour distribution.

Next, we plotted all these boxes for each field CMD on the cluster CMD and subtracted the star located closest to each box centre. Since we repeated this task for each of the two field CMD box samples, we found that some stars have remained unsubtracted once or twice. Finally, we adopted as the cluster CMD that built from stars that have not been subtracted any time. In order to illustrate the performance 
of the statistical cleaning procedure, Fig. 4 depicts a single field-star CMD (left-hand panel) for an annular region around Haffner 9 with an area equal to that of the cluster, and the cleaned cluster CMD superimposed to the observed ones represented by black and gray filled circles, respectively (right-hand panel). As can be seen, differences in stellar composition become noticeable when comparing field and cleaned cluster CMDs. Particularly, the cluster evolved MS is now clearly seen.

\subsection{Cluster's fundamental parameters}

We present in Fig. 5 the whole set of CMDs and colourcolour (CC) diagrams for Haffner 9 that can be exploited from the present multi-band photometry. They include every magnitude and colour for the whole sample of observed stars, those located within the cluster radius and those considered cluster stars from the field star decontamination procedure plotted with grey, red and black filled circles, respectively. At first glance, the cleaned cluster CMDs resemble those of a cluster with a moderate age, projected on to a star field not easy to disentangle from the cluster.

The availability of three CMDs and three different CC diagrams covering wavelengths from the blue up to the near-infrarred allowed us to derive reliable ages, reddenings and distances for Haffner 9 from the matching of theoretical isochrones. In order to enter the isochrones into the CMDs and CC diagrams we used the following ratios: $E(V-I) / E(B-V)=1.25, A_{V} / E(B-V)=3.1$ (Cardelli et al. 1989); $E\left(C-T_{1}\right) / E(B-V)=1.97$ and $A_{T_{1}} / E(B-V)$ $=2.62$ (Geisler 1996).

We started by selecting theoretical isochrones (Bressan et al. 2012) with solar metal content in order to choose that which best match the cluster's features in the CMDs. In this sense, the shape of the MS, its curvature, the relative distance between the red giants and the MS turn-off (MSTO) in magnitude and colour separately, among others, are features tightly related to the cluster age, regardless their reddenings and distances. Note also that, by considering the whole metallicity range of the Milky Way open clusters (see, e.g. Paunzen et al. 2010; Heiter et al. 2014) and by using the theoretical isochrones of Bressan et al. (2012), the differences at the zero age main sequence (ZAMS) in $V-I$ colour is smaller than $\sim 0.08 \mathrm{mag}$. This result implies that negligible differences between the ZAMSs for the cluster metallicity and that of solar metal content would appear, keeping in mind the intrinsic spread of the stars in the $V$ vs $V-I$ CMD.

From our first choices, we derived the cluster reddening by shifting those isochrones in the three CC diagrams following the reddening vectors until their bluest points coincided with the observed ones. Note that this requirement allowed us to use the three CC diagrams, even though the reddening vectors run almost parallell to the cluster sequence. Finally, the mean $E(B-V)$ colour excesse was used to properly shift the chosen isochrones in the three CMDs in order to derive the cluster true distance modulus by shifting the isochrones along the magnitude axes. We iterated this procedure for different ages as well as for metallicities $[\mathrm{Fe} / \mathrm{H}]=-0.5$ up to 0.2 dex. We found that isochrones bracketing the cluster age $\left(\log \left(t \mathrm{yr}^{-1}\right)=8.55\right)$ by $\Delta \log \left(t \mathrm{yr}^{-1}\right)= \pm 0.05$ and the cluster metallicity $([\mathrm{Fe} / \mathrm{H}]=0.0 \mathrm{dex})$ by $\Delta[\mathrm{Fe} / \mathrm{H}]= \pm 0.1$ dex represent the overall age and metallicity uncertainties owing to the observed dispersion in the cluster CMDs and $\mathrm{CC}$ diagrams. As for the cluster reddening and true distance modulus, we obtained $E(B-V)=0.60 \pm 0.05 \mathrm{mag}$ and $m-M_{o}=12.5 \pm 0.2 \mathrm{mag}$, respectively. Fig. 5 shows the adopted best matched isochrone overplotted on to the CMDs and CC diagrams with a blue solid line.

The cluster mass and its present mass function (MF) were derived by summing the individual masses of stars not eliminated during the complete cleaning procedure. Those individual masses were obtained by interpolation in the theoretical isochrone traced in Fig. 5 from the observed $T_{1}$ magnitude of each star, properly corrected by reddening and distance modulus. We finally attained $\log \left(M_{c l s} / \mathrm{M}_{\odot}\right)$ $=2.2 \pm 0.2$. The uncertainty comes from propagation of the $T_{1}$ magnitude errors in the mass distribution along the theoretical isochrone as well as from considering stars subtracted once in the field star cleaning procedure. Note that, when building the stellar density profile from stars not eliminated at any time, we obtained a curve which matches the King's curve drawn in Fig. 3. This very good agreement implies that the cleaning procedure subtracted an appropriate number of stars according to the stellar density of the backgroung/foreground field, so that the mass uncertainty would be smaller if we did not considered stars subtracted once. The resulting MF is shown in Fig. 6 where the errorbars come from applying Poisson statistics. For comparison porpuses we superimposed the relationship given by Salpeter (1955, slope $=-2.35)$ for the stars in the solar neighbourhood.

Using the resulting mass and the half-mass radius $r_{h}$, we computed the half-mass relaxation times using the equation (Spitzer \& Hart 1971):

$t_{r}=\frac{8.9 \times 10^{5} M_{c l s}^{1 / 2} r_{h}^{3 / 2}}{\bar{m} \log _{10}\left(0.4 M_{c l s} / \bar{m}\right)}$,

where $M_{c l s}$ is the cluster mass and $\bar{m}$ is the mean mass of the cluster stars $\left(\bar{m}=1.7 \mathrm{M}_{\odot}\right)$. We obtained $t_{r}=16$ $\pm 2 \mathrm{Myr}$. If we considered non-oberved stars with masses between 1 and $0.5 \mathrm{M}_{\odot}$ and the Salpeter's mass function, the relaxation times would increase in $\sim 10$ per cent. Note that, despite the advanced state of dynamical evolution of Haffner $9\left(<\right.$ age $\left./ t_{r}>=22\right)$, the cluster still keeps its $\mathrm{MF}$ close to that of Salpeter's law (see Fig. 6).

Finally, we employed the ASteCA suit of functions (Perren et al. 2015$)$ to generate $\approx 2.2 \times 10^{5}$ synthetic CMDs of a star cluster covering ages from $\log \left(t \mathrm{yr}^{-1}\right)=8.5$ up to 8.6 $\left(\Delta \log \left(t \mathrm{yr}^{-1}\right)=0.01\right)$, metallicities in the range $Z=0.012$ - $0.019(\Delta Z=0.001)$, interstellar extinction between 0.55 and $0.65 \mathrm{mag}(\Delta E(B-V)=0.01 \mathrm{mag})$, distance modulus between 12.3 and $12.7 \mathrm{mag}\left(\Delta(m-M)_{o}=0.05 \mathrm{mag}\right)$ and total mass in the range $100-250 \mathrm{M}_{\odot}\left(\Delta M=10 \mathrm{M}_{\odot}\right)$, respectively.

The steps by which a synthetic star cluster for a given set of age, metalicity, distance modulus, and reddening values is generated by ASteCA is as follows: i) a theoretical isochrone is picked up, densely interpolated to contain a thousand points throughout its entire length, including the most evolved stellar phases. ii) The isochrone is shifted in colour and magnitude according to the $E(B-V)$ and $(m-M)_{o}$ values to emulate the effects these extrinsic param- 

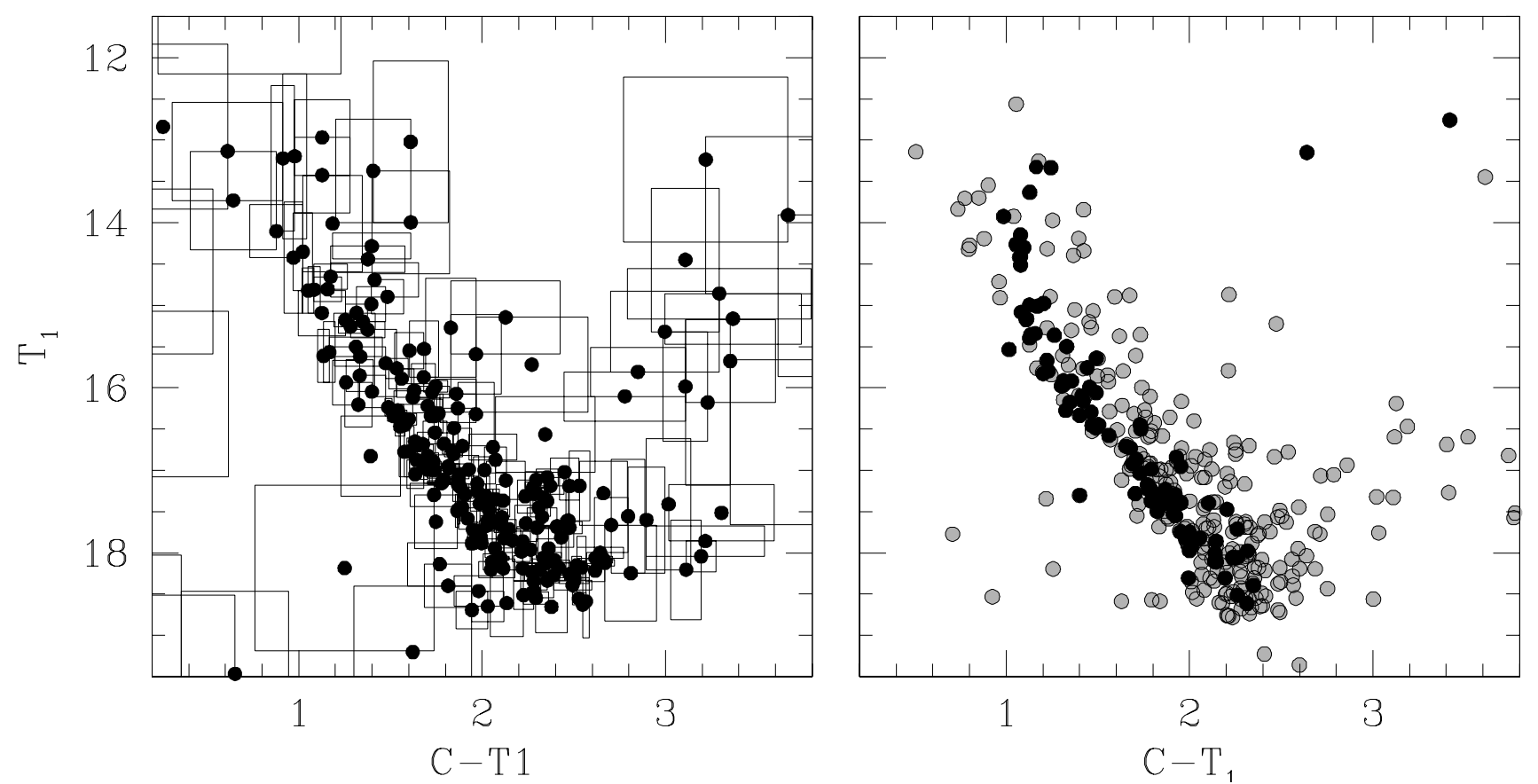

Figure 4. CMDs for stars in the field of Haffner 9: a field CMD for annular region centred on the cluster and with a size equal to the cluster area, and the corresponding defined set of boxes overplotted (left-hand panel) and; the observed and cleaned CMD composed of the stars distributed within the cluster radius represented by gray and black filled circles, respectively (left-hand panel).

eters have over the isochrone in the CMD. iii) The isochrone is trimmed down to a certain faintest magnitude according to the limiting magnitude thought to be reached. iv) An initial mass function (IMF) is sampled in the mass range $[\sim 0.01-100] M_{\odot}$ up to a total mass value $M$ provided that evolved CMD regions result properly populated. The distribution of masses is then used to obtain a properly populated synthetic star cluster by keeping one star in the interpolated isochrone for each mass value in the distribution. v) A random fraction of stars are assumed to be binaries, which is set by default to $50 \%$ (von Hippel 2005), with secondary masses drawn from a uniform distribution between the mass of the primary star and a fraction of it given by a mass ratio parameter set to 0.7. vi) An appropriate magnitude completeness and an exponential photometric error functions are finally applied to the synthetic star cluster.

Fig. 7 shows the synthetic CMD which best matches the cluster's parameters, with the generated uncertainties in $T_{1}$ and $C-T_{1}$, the range of stellar masses drawn in colourscaled filled circles and the theoretical isochrone for $\log (t$ $\left.\mathrm{yr}^{-1}\right)=8.55$ and $[\mathrm{Fe} / \mathrm{H}]=0.0$ dex superimposed.

\section{ANALYSIS AND DISCUSSION}

Dias et al. (2014) presented a catalog of mean proper motions and membership probabilities of individual stars for optically visible open clusters, among them, Haffner 9, using data from the UCAC4 catalog (Zacharias et al. 2013). We cross-correlated the stars with proper motions in our data sets and built the corresponding $T_{1}$ vs. $C-T_{1}$ CMD. The left-hand panel of Fig. 8 shows with grey, red and black filled circles all these stars, those with membership probabilities $P>75$ and 90 per cent, respectively. As can be seen, the proper motion memberships confirm that the cleaned CMD produced in Section 3.2, which is very well matched by an isochrone of $\log \left(t \mathrm{yr}^{-1}\right)=8.55$ and $[\mathrm{Fe} / \mathrm{H}]=0.0$ dex (see details in Section 3.3), corresponds to that of the cluster. This is an important probe, since previous detailed photometric studies of this object led to different fundamental parameters.

For instance, Bica \& Bonatto (2005) and Kharchenko et al. (2013) used 2MASS data (Skrutskie et al. 1997), separately, and derived an age of $140 \pm 20 \mathrm{Myr}$, a true distance modulus $(m-M)_{o}=11.40 \pm 0.1 \mathrm{mag}$, and a reddening $E(B-V)=0.50 \pm 0.05$ from the fit of theoretical isochrones in the $J$ versus $J-H$ CMD. However, Buckner \& Froebrich (2014), also from 2MASS data, obtained an age of $250 \pm 100$ Myr, $(m-M)_{o}=12.40 \pm 0.4 \mathrm{mag}$, and $E(B-V)=0.40$ \pm 0.10 , respectively. All three studies assumed a solar metal content. In order to seek for any source of discrepancy in these 2MASS data analyses, we took advantage of the photometric and proper motion membership probabilities analyzed above. Fig. 8 (middle and right-hand panels) depict the 2MASS CMDs used by Bica \& Bonatto (2005) and Buckner \& Froebrich (2014) to derive the cluster's fundamental parameters. We plotted every star with proper motion using the same colour code as in the left-hand panel. Note that the fainter magnitude limit reached by stars with proper motion measurements is nearly similar to that of the 2MASS data. For the sake of the reader, we superimposed the isochrone of $\log \left(t \mathrm{yr}^{-1}\right)=8.55$ and $[\mathrm{Fe} / \mathrm{H}]=0.0$ dex as well as those for the reddenings, distance modulii, ages and metallicities 

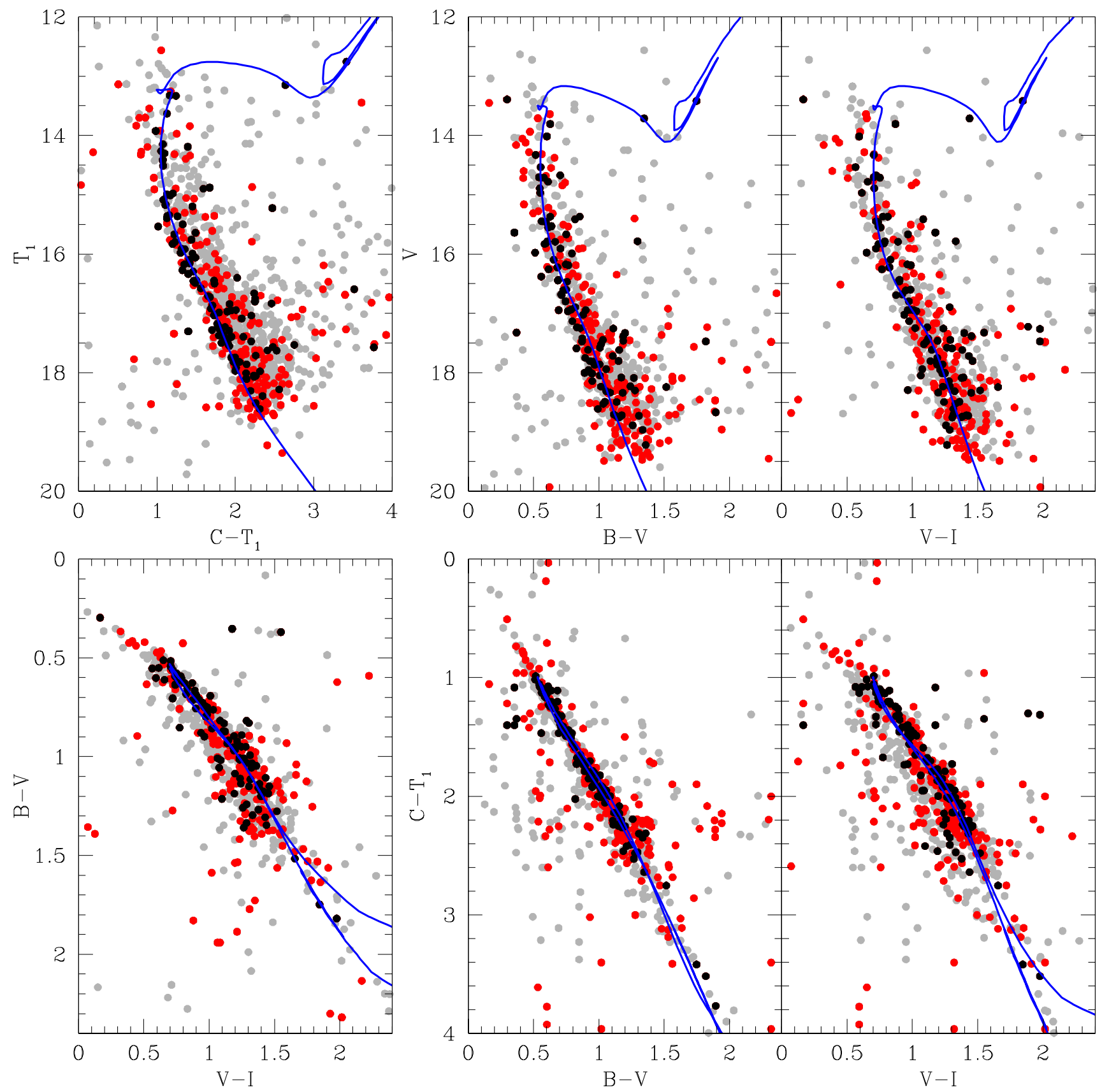

Figure 5. CMDs and CC diagrams for stars measured in the field of Haffner 9. Grey, red and black filled circles represent all the measured stars, those within the cluster radius and the cluster stars from the field star cleaning procedure, respectively. We overplotted the isochrone which best matches the cluster features (see text for details).

used by Bica \& Bonatto (2005) and Buckner \& Froebrich (2014) with blue and magenta solid lines, respectively.

From the examination of these CMDs some conclusions can be drawn. Firstly, the magnitude depth of 2MASS data is noticeable much brigher than that of the present data set (see Fig. 5), whereas the colour baseline of the infrared colours is much shorter than that of $C-T_{1}$, which do not favour an accurate isochrone matching. Secondly, the isochrone adopted in this work reasonably matches the cluster sequences, although the scatter is larger than that seen in the $T_{1}$ vs. $C-T_{1}$ CMD. Seemingly, Bica \& Bonatto (2005) have fitted a MS with some field contamination (middle panel). In the case of Buckner \& Froebrich (2014) we especulate with the possibility that they could have considered a larger cluster area with field stars with bluer infrared colours and no proper motions.

Hasegawa et al. (2008) presented BVI photometry of stars in the cluster field and estimated an age of $500 \mathrm{Myr}$, a metal content $[\mathrm{Fe} / \mathrm{H}]=-0.4 \mathrm{dex}$, a true distance modulus of $(m-M)_{o}=12.7 \mathrm{mag}$, and a reddening $E(B-V)=0.75 \mathrm{mag}$. 


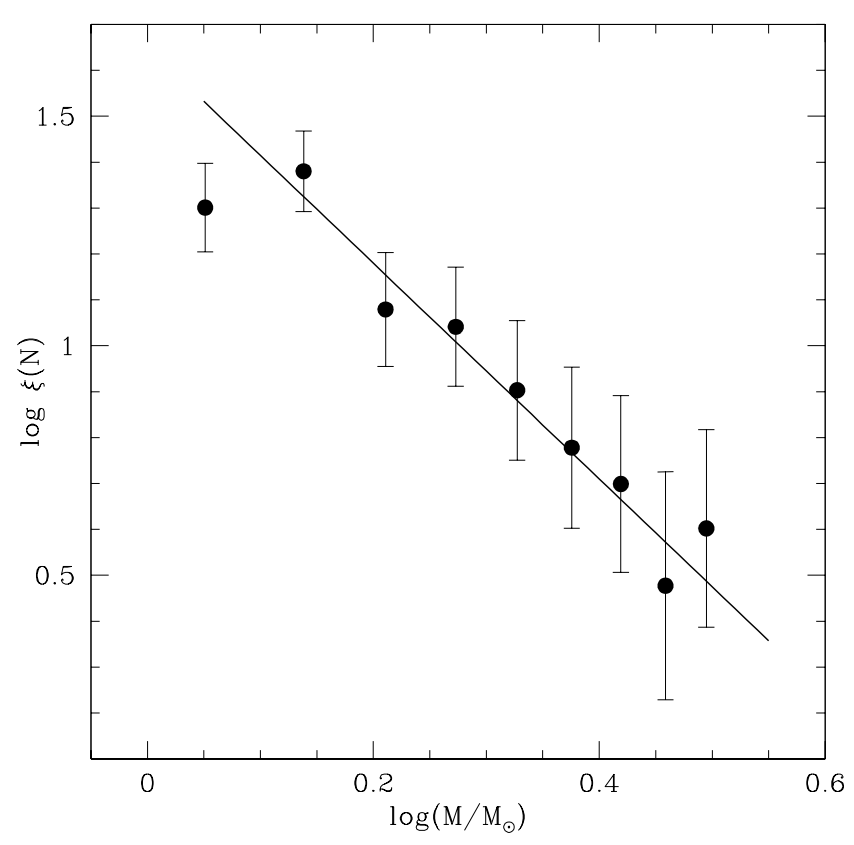

Figure 6. The present mass function of Haffner 9. The Salpeter (1955)' relationship for stars in the solar neighbourhood is superimposed with a solid line.

For comparison purposes, we plotted such an isochrone in the left-hand panel of Fig. 8 with a magenta solid line. Once again, the employment of a field star contaminated CMD could lead them to derive unreliable cluster parameters.

We computed Galactic coordinates using the derived cluster heliocentric distances, their angular Galactic coordinates and a Galactocentric distance of the Sun of $\mathrm{R}_{G C_{\odot}}=$ $8.3 \mathrm{kpc}$ (Hou \& Han 2014, and references therein). The resulting spatial distribution is depicted in the top-left panel of Fig. 9, where we added for comparison purposes the 2167 open clusters catalogued by Dias et al. (2002, version 3.5 as of January 2016) and the schematic positions of the spiral arms (Drimmel \& Spergel 2001; Moitinho et al. 2006). Haffner 9 is located at the Perseus arm, beyond the cirle around the Sun $(\mathrm{d} \sim 2.0 \mathrm{kpc})$ where the catalogued clusters are mostly concentrated.

The age $/ t_{r}$ ratio is a good indicator of the internal dynamical evolution, since it gives the number of times the characteristic time-scale to reach some level of energy equipartition (Binney \& Merrifield 1998) has been surpassed. Star clusters with large age $/ t_{r}$ ratios have reached a higher degree of relaxation and hence are dynamically more evolved. As Fig. 9 shows, Haffner 9 appears to have had enough time to evolve dynamically. In the figure we included in grey colour 236 open clusters analysed by Piskunov et al. (2007), who derived from them homogeneous scales of radii and masses. They derived core and tidal radii for their cluster sample, from which we calculated the half-mass radii and, with their clusters masses and eq. 4, relaxation times, by assuming that the cluster stellar density profiles can be indistinguishably reproduced by King and Plummer models. Their cluster sample are mostly distributed inside a circle of $\sim 1 \mathrm{kpc}$ from the Sun. As compared to the Piskunov

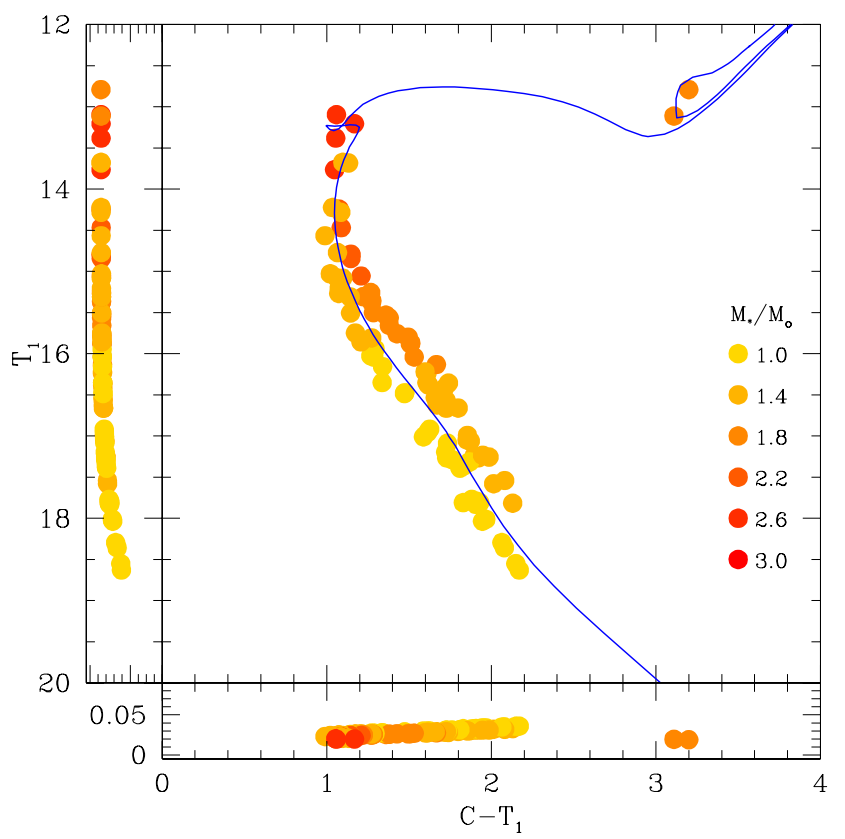

Figure 7. Best-generated star cluster CMD with the uncertainties in $T_{1}$ and $C-T_{1}$, the stellar masses in colour-scaled filled circles, and the theoretical isochrone for $\log \left(t \mathrm{yr}^{-1}\right)=8.55$ and $[\mathrm{Fe} / \mathrm{H}]=0.0$ dex superimposed.

et al. (2007)'s sample, Haffner 9 is placed towards the most evolved limit of the age $/ t_{r}$ distribution (right-hand panels).

Since dynamical evolution implies the loss of stars (mass loss), we expect some trend of the present-day cluster mass with the age $/ t_{r}$ ratio. This is confirmed in the top-right panel of Fig. 5, where the larger the present-day mass the less the dynamical evolution of a cluster in the solar neighbourhood, with a noticeable scatter. Haffner 9 appears to have a relatively large mass for its particular internal dynamical state. Curiously, selection against poor and old clusters could suggest the beggining of cluster dissolution, with some exceptions.

Trenti et al. (2010) presented a unified picture for the evolution of star clusters on the two-body relaxation timescale from direct $\mathrm{N}$-body simulations of star clusters in a tidal field. Their treatment of the stellar evolution is based on the approximation that most of the relevant stellar evolution occurs on a timescale shorter than a relaxation time, when the most massive stars lose a significant fraction of mass and consequently contribute to a global expansion of the system. Later in the life of a star cluster, two-body relaxation tends to erase the memory of the initial density profile and concentration. They found that the structure of the system, as measured by the core to half mass radius ratio, the concentration parameter $c=\log \left(r_{t} / r_{c}\right)$, among others, evolve toward a universal state, which is set by the efficiency of heating on the visible population of stars induced by dynamical interactions in the core of the system. In the bottom panels of Fig. 9 we plotted the dependence of the concentration parameter $c$ with the cluster mass and the age $/ t_{r}$ ratio, respectively. They show that our dynamically evolved cluster is within those with relatively high $c$ values, and that star clusters tend to initially start their dynam- 
ical evolution with relatively small concentration parameters. Likewise, star clusters in an advanced dynamical state can also have relatively lower $c$ values due to their smaller masses.

\section{SUMMARY AND CONCLUSIONS}

In order to continue our Washington $C T_{1}$ and Johnson $B V I$ photometric studies on Milky Way star clusters, we turned our attention to Haffner 9, a previously studied open cluster with a noticeable spread in the values obtainted of its fundamental parameters.

The analysis of the current photometric data sets leads to the following main conclusions:

(i) To disentagle cluster features from those belonging to their surrounding fields, we applied a subtraction procedure to statistically clean the cluster CMDs from field star contamination. The employed technique makes use of variable cells in order to reproduce the field CMD as closely as possibly. The stellar density profile built from stars that reamined unsubtracted very well matches that obtained from star counts carried out throughout the observed field, once the background level is subtracted. Moreover, the main cluster features in the cleaned CMDs are confirmed when proper motion membership probabilities are taken into account.

(ii) Using the cleaned cluster CMDs and CC diagrams, we estimated the cluster fundamental parameters in a selfconsistent way. The availability of three $\mathrm{CC}$ diagrams and three CMDs covering wavelengths from the blue up to the near-infrarred allowed us to derive reliable values of age, metallicity, reddenings and distances for Haffner 9. We exploited such a wealth in combination with theoretical isochrones computed by Bressan et al. (2012) to find out that the cluster is $350 \mathrm{Myr}$ old, is placed in the Perseus arm at a solar heliocentric distance of $3.2 \mathrm{kpc}$, and has nearly solar metal content. The lower limits of its present mass is $\sim 160 \mathrm{M}_{\odot}$. We confirmed such a limit from the generation of thousand synthetic CMDs.

(iii) We found that a less deep photometry, a narrower colour baseline and a less effective CMD cleaning procedure, could have been some sources that led previous studies to derive cluster fundamental parameters which do not correspond to the fiducial cluster features. By using the same photometric data sets as in previous works and proper motion memberships, we confirm the present cluster fundamental parameters.

(iv) Finally, we estimated the half-mass relataxion time for Haffner 9, which turned out to be $\sim 22$ smaller than the cluster age. This result suggests that Haffner 9 is facing an advanced state of its internal dynamical evolution. However, the cluster still keeps its MF close to that of the Salpeter's law. When combined with the obtained structural parameters, we found that the cluster is possibly in the phase typical of those with mass segregation in their core regions. whose thorough comments and suggestions allowed us to improve the manuscript.

\section{REFERENCES}

Bica E., Bonatto C., 2005, A\&A, 443, 465

Binney J., Merrifield M., 1998, Galactic Astronomy

Bonatto C., Bica E., 2007, MNRAS, 377, 1301

Bressan A., Marigo P., Girardi L., Salasnich B., Dal Cero C., Rubele S., Nanni A., 2012, MNRAS, 427, 127

Buckner A. S. M., Froebrich D., 2014, MNRAS, 444, 290

Canterna R., 1976, AJ, 81, 228

Cardelli J. A., Clayton G. C., Mathis J. S., 1989, ApJ, 345, 245

Carraro G., Beletsky Y., Marconi G., 2013, MNRAS, 428, 502

Dias W. S., Alessi B. S., Moitinho A., Lépine J. R. D., 2002, A\&A, 389, 871

Dias W. S., Monteiro H., Caetano T. C., Lépine J. R. D., Assafin M., Oliveira A. F., 2014, A\&A, 564, A79

Drimmel R., Spergel D. N., 2001, ApJ, 556, 181

Geisler D., 1996, AJ, 111, 480

Hasegawa T., Sakamoto T., Malasan H. L., 2008, PASJ, 60, 1267

Heiter U., Soubiran C., Netopil M., Paunzen E., 2014, A\&A, 561, A93

Hou L. G., Han J. L., 2014, A\&A, 569, A125

Kharchenko N. V., Piskunov A. E., Schilbach E., Röser S., Scholz R.-D., 2013, A\&A, 558, A53

King I., 1962, AJ, 67, 471

Lauberts A., 1982, ESO/Uppsala survey of the $\operatorname{ESO}(\mathrm{B})$ atlas

Lindsay E. M., 1958, MNRAS, 118, 172

Maia F. F. S., Corradi W. J. B., Santos Jr. J. F. C., 2010, MNRAS, 407, 1875

Moitinho A., Vázquez R. A., Carraro G., Baume G., Giorgi E. E., Lyra W., 2006, MNRAS, 368, L77

Paunzen E., Heiter U., Netopil M., Soubiran C., 2010, A\&A, 517, A32

Pavani D. B., Bica E., 2007, A\&A, 468, 139

Perren G. I., Vázquez R. A., Piatti A. E., 2015, A\&A, 576, A6

Piatti A. E., Bica E., 2012, MNRAS, 425, 3085

Piatti A. E., Sarajedini A., Geisler D., Gallart C., Wischnjewsky M., 2007, MNRAS, 382, 1203

Piskunov A. E., Schilbach E., Kharchenko N. V., Röser S., Scholz R.-D., 2007, A\&A, 468, 151

Plummer H. C., 1911, MNRAS, 71, 460

Salpeter E. E., 1955, ApJ, 121, 161

Skrutskie M. F., et al., 1997, in Garzon F., Epchtein N., Omont A., Burton B., Persi P., eds, Astrophysics and Space Science Library Vol. 210, The Impact of Large Scale Near-IR Sky Surveys. p. 25, doi:10.1007/978-94-011-5784-14

Spitzer Jr. L., Hart M. H., 1971, ApJ, 164, 399

Stetson P. B., Davis L. E., Crabtree D. R., 1990, in Jacoby G. H., ed., Astronomical Society of the Pacific Conference Series Vol. 8, CCDs in astronomy. pp 289-304

Trenti M., Vesperini E., Pasquato M., 2010, ApJ, 708, 1598

Zacharias N., Finch C. T., Girard T. M., Henden A., Bartlett J. L., Monet D. G., Zacharias M. I., 2013, AJ, 145, 44 von Hippel T., 2005, ApJ, 622, 565

This paper has been typeset from a $\mathrm{T}_{\mathrm{EX}} / \mathrm{LAT}_{\mathrm{EX}}$ file prepared by the author.

\section{ACKNOWLEDGEMENTS}

We thank Giovanni Carraro for revising the manuscript and making useful suggestions. We thank the anonymous referee 

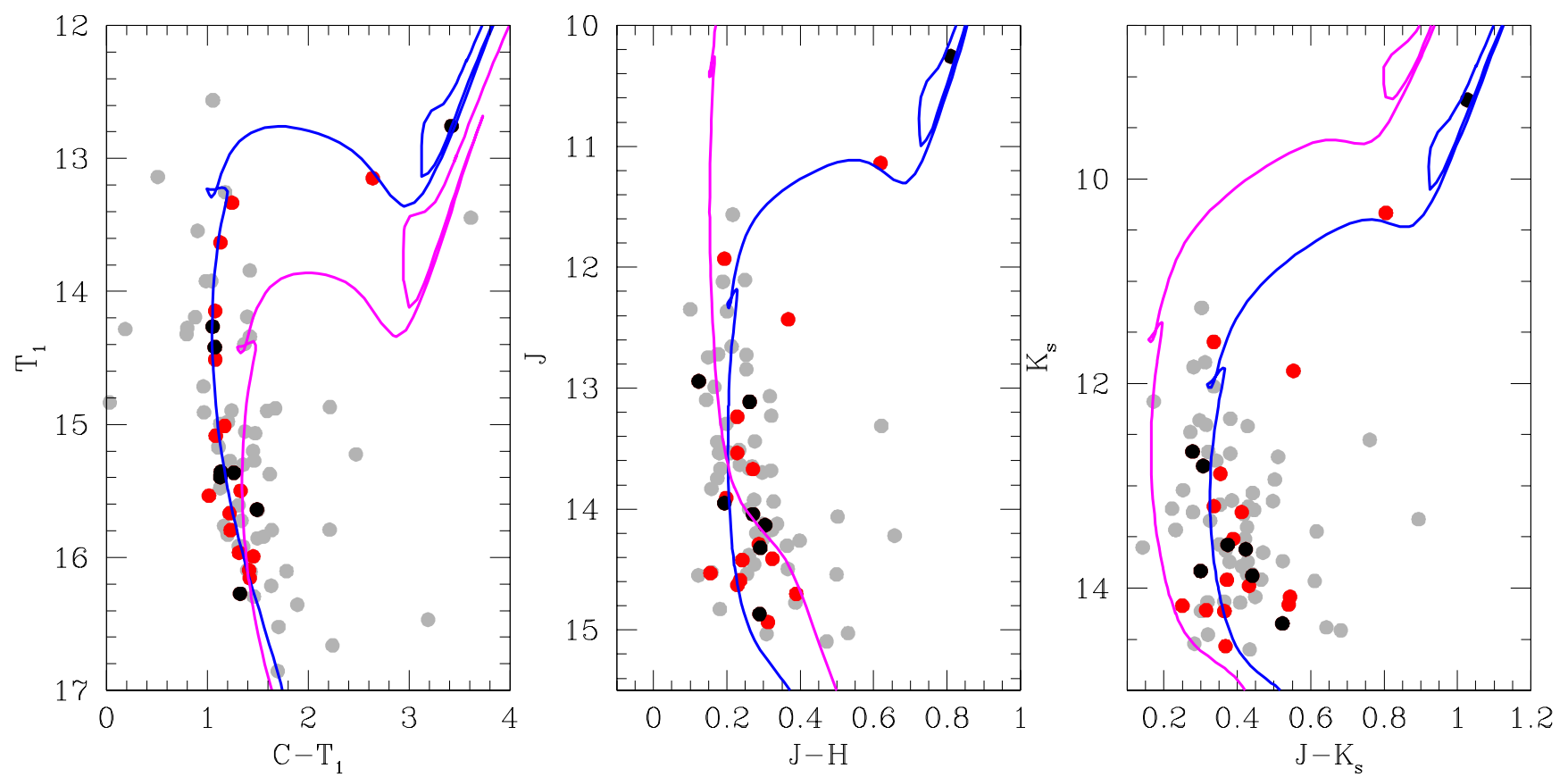

Figure 8. CMDs for stars in the field of Haffner 9 with proper motion measurements (grey filled circles). Stars with proper motion membership probabilities higher than 75 and 90 per cent are drawn with red and black filled circles, respectively. The isochrones adopted in this work is superimposed with a blue solid line, while those for the parameters estimated by Hasegawa et al. (2008), Bica \& Bonatto (2005) and Buckner \& Froebrich (2014) are plotted with a magenta line in the left-hand, middle and right-hand panels, respectively (see text in Section 4 for details. 


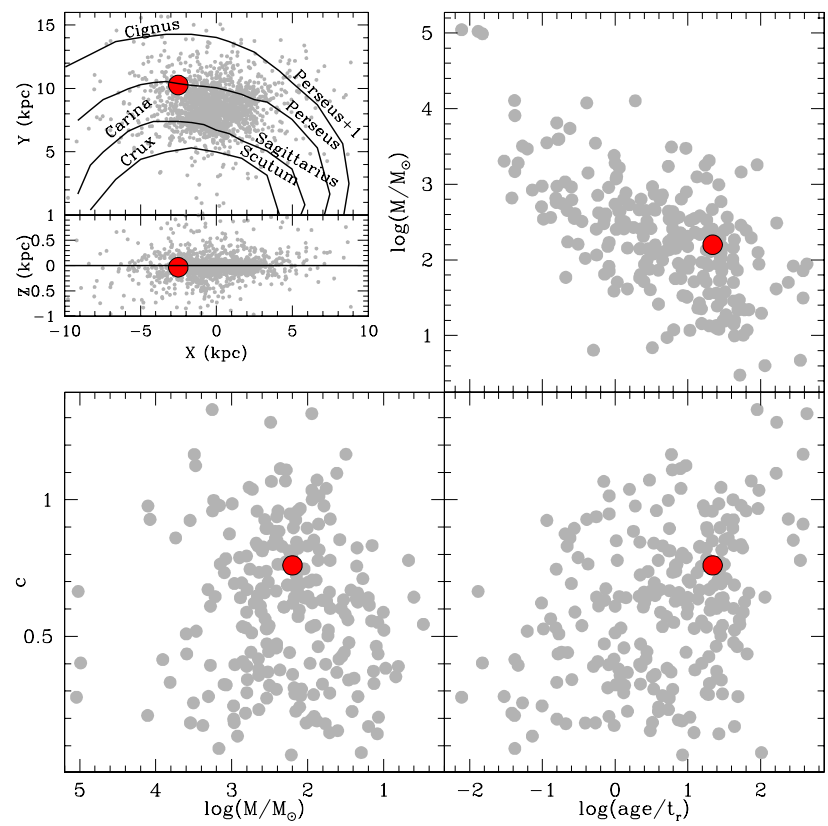

Figure 9. Top-left: Galactic spatial position of Haffner 9 (red filled cirled). Open clusters from the catalogue of Dias et al. (2002, version 3.5 as of January 2016) are drawn with gray dots, while the schematic positions of spiral arms (Drimmel \& Spergel 2001; Moitinho et al. 2006) are traced with black solid lines. Top-right and bottom: Relationships between cluster concentration parameter $(c)$, mass, age and relaxation time $\left(t_{r}\right)$. Grey dots correspond to 236 star clusters with homogeneous estimations of masses and radii derived by Piskunov et al. (2007). 